\title{
Obtención de biodiesel a partir de grasa bovina
}

\author{
Pablo Arévalo*, José Ulloa, Servio Astudillo
}

Carrera de Ingeniería Ambiental, Universidad Politécnica Salesiana, Cuenca, Ecuador.

* Autor para correspondencia : parevalo@ups.edu.ec

\section{Resumen}

En este trabajo se estudia específicamente la reacción de esterificación de los ácidos grasos saturados de bajo valor comercial con alcoholes de cadena corta, como el metanol anhidro al $95 \%$ y metanol comercial para la elaboración de biodiesel. En las experiencias realizadas se estudiaron las condiciones óptimas de reacción, variando la relación peso alcohol-catalizador $(\mathrm{KOH})$, la temperatura promedio de reacción y velocidad de adición del catalizador, que se presentan detalladamente en el artículo. Se obtiene así un biodiesel con un rendimiento entre el 88 y $90 \%$ con una concentración de catalizador del 3,7 a $3,8 \%$ a una temperatura de $58^{\circ} \mathrm{C}$. Además se determinó que el biodiesel logrado cumple con los estándares internacionales y que disminuye las emisiones tanto de $\mathrm{SO}_{2}$ como el $\mathrm{CO}_{2}$, a pesar de que las emisiones de $\mathrm{NO}_{2}$ aumentan.

Palabras clave: Biodiesel, ácidos grasos,esterificación.

\section{Introducción}

El biodiesel es un combustible sustituto del gas oil para motores diesel obtenido de materias primas agrícolas como aceites vegetales, residuos grasos de faenamiento de animales y que presenta ventajas frente al diesel derivado del petróleo ya que se obtiene partir de fuentes renovables, como los aceites y grasas vegetales $o$ animales de cualquier tipo.

La utilización del biodiesel lleva a un balance óptimo en el ciclo de carbono, evitando la acumulación de dióxido de carbono en el medio ambiente. Estas emisiones se reducen en comparación con el diesel, pero lo más relevante es su bajo contenido de azufre, por lo que se convierte en la mejor opción al ser usado como aditivo en el diesel, $y$ así cumplir con las regulaciones ambientales que se van imponiendo cada vez en más países. Inclusive, hoy por hoy, en muchos países del mundo, existen legislaciones que imponen el uso obligatorio del biodiesel en mezcla con el gasoil.
En un sentido amplio, el biodiesel implica tres grupos diferentes de reacciones según un éster reacciona con un alcohol (transesterificación), con un ácido carboxílico (acidólisis) o con otro éster (interesterificación). El presente trabajo de investigación comprende el estudio de la metanolisis de grasas para obtener ésteres metílicos de ácidos grasos. En este sentido los términos transesterificación o alcoholisis hacen referencia a este tipo de reacción.

La reacción de transesterificación de una grasa animal es aquella en la cual, una molécula de triglicéridos, componente mayoritario en una grasa, reacciona con un alcohol, generalmente ligero, bajo la acción de un catalizador, para producir una mezcla de ésteres de ácidos grasos y glicerina. Esta reacción de grasas animales con alcoholes ligeros consta de tres etapas consecutivas reversibles en las que el triglicérido es convertido consecutivamente en digliceridos, monogliceridos y glicerina. 
La reacción de transesterificación de grasas animales con alcoholes ligeros se ha de llevar a cabo bajo la acción de un catalizador que puede ser homogéneo ácido, básico o heterogéneo; para este trabajo de investigación se utilizó un catalizador básico: el hidróxido de potasio. "Para el mecanismo de reacción bajo un catalizador básico el primer paso es la reacción entre el catalizador y el alcohol, para producir un alcóxido. El ataque nucleofílico del alcóxido sobre el grupo carbonilo del triglicérido genera un tetraedro como intermedio, del que se genera una molécula de éster de ácido graso y el correspondiente anión del diglicérido. El último paso es la desprotonación del catalizador regenerándose y reaccionando con otra molécula de alcohol y comenzando el ciclo. Los di y monoglicéridos son convertidos en una mezcla de ácidos grasos y glicerina por el mismo mecanismo descrito" (Montero, 2002-2003).

\section{Materiales y métodos}

\section{I Reactivos}

Los reactivos empleados en la elaboración del presente trabajo pueden clasificarse en tres grupos diferenciados: los reactivos para la síntesis de ésteres metílicos de ácidos grasos, el catalizador utilizado en dicha síntesis; $y$, por último, otros compuestos generales utilizados en la experimentación.

Los reactivos necesarios en la síntesis de ésteres metílicos incluyen al sebo que se obtuvo a través del proceso de fundición que separa los tejidos y elimina el agua de los residuos grasos de las tercenas de la ciudad, trabajo realizado de manera artesanal. La composición de este sebo obtenido se presenta en la Tabla I.

Tabla I. Composición del sebo después del proceso de fundición.

\begin{tabular}{lr}
\hline Componente & $\%$ \\
\hline I2-metil-ácido tridecanoico & 2,94 \\
Ácido pentanoico & 1,17 \\
9-ácido-hexadecenoico & 2,00 \\
I4 metil ácido pentadecanoico & 18,49 \\
Ácido heptadecanoico & 2,17 \\
9-ácido octadecenoico & 25,83 \\
8-ácido octadecenoico & 9,33 \\
Ácido octadecanoico (Ácido esteárico) & 28,82 \\
\hline
\end{tabular}

Otro reactivo es el metanol $\mathrm{CH}_{3} \mathrm{OH}$, que tiene un peso molecular de $32,04 \mathrm{~g} / \mathrm{mol}$, con una densidad relativa de $0,791 \%$ y una riqueza del $95 \%$. Presenta una temperatura de ebullición de $64,5^{\circ} \mathrm{C}$, fue suministrado por los laboratorios Merck.

Los distintos experimentos se llevaron a cabo utilizando un catalizador básico, como es el hidróxido de potasio $\mathrm{K}(\mathrm{OH})$, de un peso molecular de $56, \mathrm{II}$ [g/mol] y una riqueza del $90 \%$, asimismo este insumo fue suministrado por los laboratorios Merck.

En el análisis por cromatografía de capa fina se ha utilizado hexano, éter dietílico y ácido acético glacial. Para la determinación del índice de acidez, se utiliza una solución etanólica de hidróxido de potasio, como reactivo valorante, previamente con hidrógeno aftalato de potasio como patrón primario y fenolftaleína como indicador.

\subsection{Métodos utilizados}

\subsection{Análisis por Cromatografía de Capa Fina}

El análisis por cromatografía de capa fina es el método analítico empleado para analizar las muestras de ésteres metílicos de ácidos grasos. Las placas cromatográficas están hechas de aluminio cubiertas de gel de sílice, sus dimensiones son $25 \mathrm{~cm} \times 3 \mathrm{~cm}$ de las cuales se han realizado recortes de $8 \mathrm{~cm} \times 2 \mathrm{~cm}$, tamaño suficiente como para poder analizar las muestras de ésteres metílicos.

Los reactivos utilizados para esta comatografía son hexano con una riqueza del $85 \%$, éter dietílico al $15 \%$, y ácido acético glacial con una riqueza del $100 \%$, todos esos insumos han sido facilitados por laboratorios Merck. 


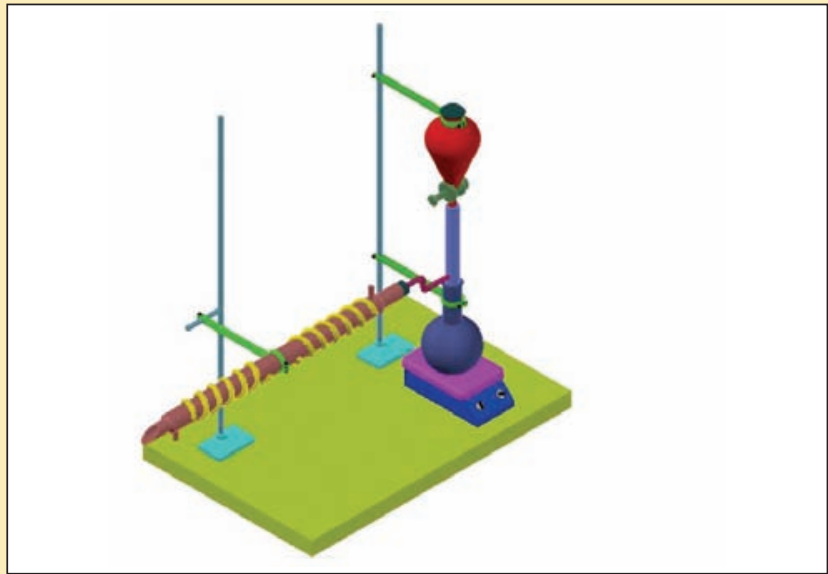

Figura I. Montaje del reactor que incluye agitador magnético y calentador, balón de destilación, refrigerante, soportes metálicos y una termocupla para determinar la temperatura.

\subsection{2 Índice de acidez y grado de acidez}

Para llevar a cabo la determinación del índice de acidez o grado de acidez de los aceites vegetales empleados, se utiliza el método descrito en la norma ISO 660-1983, donde se define al índice de acidez como los miligramos de hidróxido de potasio necesarios para neutralizar los ácidos grasos libres contenidos en un gramo de muestra.Y al grado de acidez como el porcentaje en peso de ácidos grasos libres.

\subsection{Materiales}

El siguiente esquema (Figura I) da a conocer el montaje experimental para llevar a cabo la reacción de transesterificación del sebo. Se utilizó un reactor discontinuo de vidrio, de forma cilíndrica, fondo plano y una capacidad instalada de $1 \mathrm{l}$ asentado sobre un calentador $y$ agitador magnético cuya función es facilitar el mezclado de los reactivos. En la tapa del reactor se disponen dos bocas, una para el acople de alimentación del metóxido y un controlador digital de temperatura, y la segunda para la conexión al condensador que sirve para evitar los escapes de alcohol por evaporación.
2.4 Procedimiento para el estudio de la transesterificación de la grasa..

Una vez montada la instalación experimental, se cargó la grasa en estado líquido dentro del reactor, fijando así la temperatura de reacción en el termómetro de contacto y se preparó la disolución del catalizador en metanol. Alcanzada la temperatura de reacción se conectó el refrigerante de reflujo y se puso en marcha el sistema de agitación. Finalmente se añadió al reactor la disolución de metóxido. Este será el inicio de cada experimento, es decir, tiempo cero.

Después de este paso, se dejó transcurrir el tiempo de reacción por una hora y se procedió a separar las fases obtenidas utilizando un embudo de separación, se determinó el $\mathrm{pH}$ y el volumen obtenido de la fase clara y se realizó el análisis de cromatografía de capa fina para comprobar la presencia de ésteres metílicos de ácidos grasos. Por último se recuperó el exceso de alcohol a través del proceso de destilación.

\section{Resultados}

\section{I Estudio de la influencia de la cantidad de catali- zador.}

El objetivo fundamental de esta etapa es determinar la cantidad mínima de hidróxido de potasio en la que se obtiene la conversión total hacia ésteres metílicos de ácidos grasos. Para obtener este valor se procede experimentalmente según la reacción de transesterificación, bajo una temperatura de $58^{\circ} \mathrm{C}$, en un tiempo de reacción de I hora; los volúmenes de alcohol y de grasa son de 250 cc y 500 cc respectivamente, y la agitación debe darse a 1000 rpm.

Se procedió a variar la cantidad de catalizador de $0,5 \mathrm{~g}$, obteniendo los resultados presentados en la Tabla 2.

Tabla 2. Resultados de la $1^{\circ}$ Fase.

\begin{tabular}{cccc}
\hline CATALIZADOR & ESTER METÍLICO & $\mathrm{PH}$ \\
\hline $2,0 \mathrm{~g}$ & $568 \mathrm{cc}$ & $566 \mathrm{cc}$ & 7,0 \\
$2,5 \mathrm{~g}$ & $600 \mathrm{cc}$ & $604 \mathrm{cc}$ & $7, \mathrm{I}$ \\
$3,0 \mathrm{~g}$ & $550 \mathrm{cc}$ & $55 \mathrm{Icc}$ & 7,5 \\
$3,5 \mathrm{~g}$ & $582 \mathrm{cc}$ & $580 \mathrm{cc}$ & 7,8 \\
$4,0 \mathrm{~g}$ & $544 \mathrm{cc}$ & $540 \mathrm{cc}$ & 7,9 \\
$4,5 \mathrm{~g}$ & $548 \mathrm{cc}$ & $545 \mathrm{cc}$ & 8,0 \\
$5,0 \mathrm{~g}$ & $531 \mathrm{cc}$ & $528 \mathrm{cc}$ & 9,3 \\
$6,5 \mathrm{~g}$ & $554 \mathrm{cc}$ & $550 \mathrm{cc}$ & 11,4 \\
\hline
\end{tabular}


Al realizar el análisis del modelo de regresión a utilizarse se estableció que el más conveniente era el determinado por la siguiente ecuación racional (Ecuación I).

$$
y=\frac{1}{\left(a+b x^{2}\right)}
$$

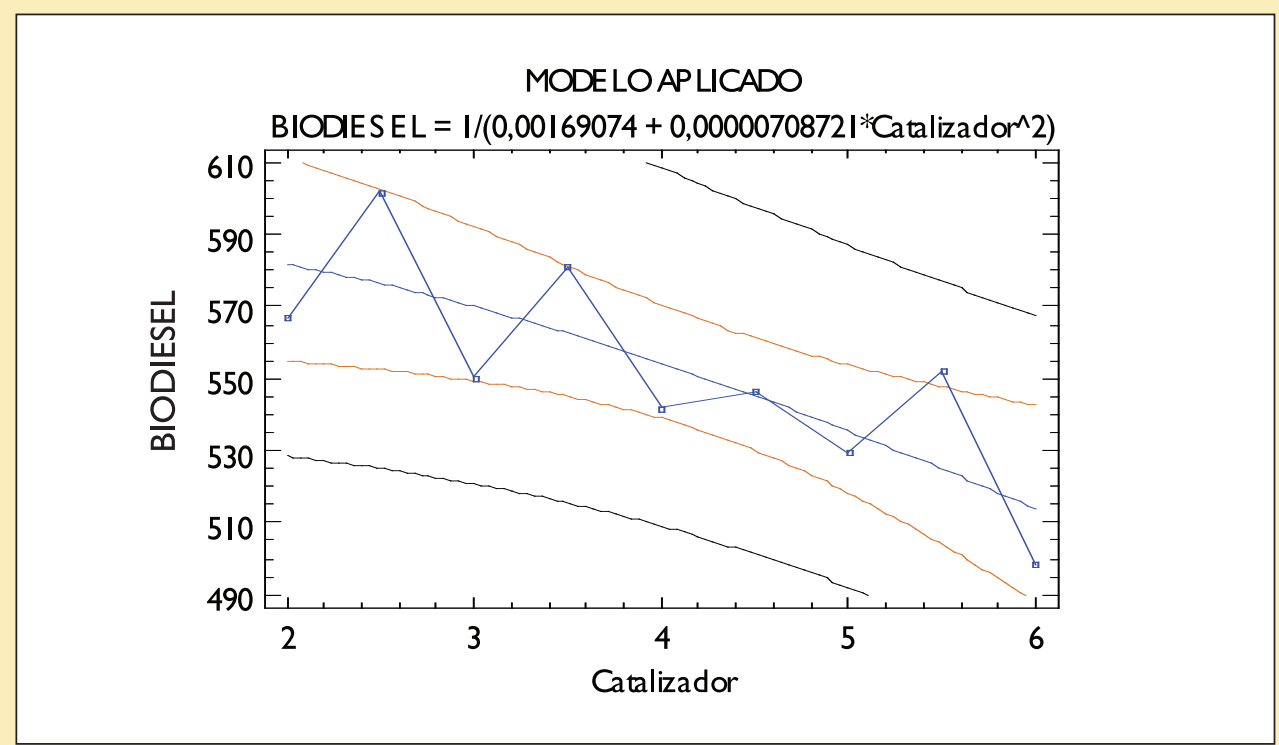

Figura 2. Modelo alcanzado que relaciona la Cantidad de Biodisel en [cc] en función de los gramos de Catalizador.

\subsection{Optimización de la reacción}

El objetivo en esta segunda fase es la optimización de la reacción de ésteres metílicos, obtenidos a partir grasa bovina en reactores discontinuos, para ello, se ha empleado la metodología del diseño factorial de experimentos y superficies de respuesta.

Se inicia con la elección de la variable a medir que es rendimiento de la fase éster, en tanto por ciento en peso, a la hora de reacción, además se determinan las variables de operación: temperatura y la concentración inicial de catalizador.

La velocidad de agitación se ha mantenido constante en todos y cada uno de los experimentos realizados, de forma que ésta fuese elevada a $1000 \mathrm{rpm}$ para que no tengan lugar las posibles limitaciones de transferencia de materia en el medio de reacción, con lo que se eliminan los efectos de difusión, y la única etapa controlante es la reacción química. Así mismo, se han llevado a cabo los experimentos a presión atmosférica.

Además, se determinaron intervalos de operación para cada una de las variables de operación: elegidos en función de la permisividad del equipo y
Que dio el resultado presentado a continuación (Figura 2). Así, en esta fase se proyecta y considera como puntos a trabajar los valores de 2-2,5-3 gramos de catalizador. 
Tabla 3. Experimento correspondiente al diseño experimental.

\begin{tabular}{ccc}
\hline $\begin{array}{c}\text { Temperatura } \\
{ }^{\circ} \mathrm{C}\end{array}$ & $\begin{array}{c}\text { Concentración } \\
\% \mathrm{P} / \mathrm{P}\end{array}$ & $\begin{array}{c}\text { Rendimiento } \\
\% \mathrm{P} / \mathrm{P}\end{array}$ \\
\hline 58,0 & 0,36 & 85,7 \\
58,0 & 0,36 & 86,7 \\
58,0 & 0,36 & 85,7 \\
58,0 & 0,36 & 86 \\
56,0 & 0,28 & 81,1 \\
60,0 & 0,43 & 78,6 \\
60,0 & 0,28 & 80 \\
56,0 & 0,43 & 78 \\
\hline
\end{tabular}

Como se muestra en la gráfica (Figura 3), se aprecia que el rendimiento de la reacción aumentó con la temperatura en una concentración alta, pero que disminuye cuando aumentamos la temperatura con una concentración menor.

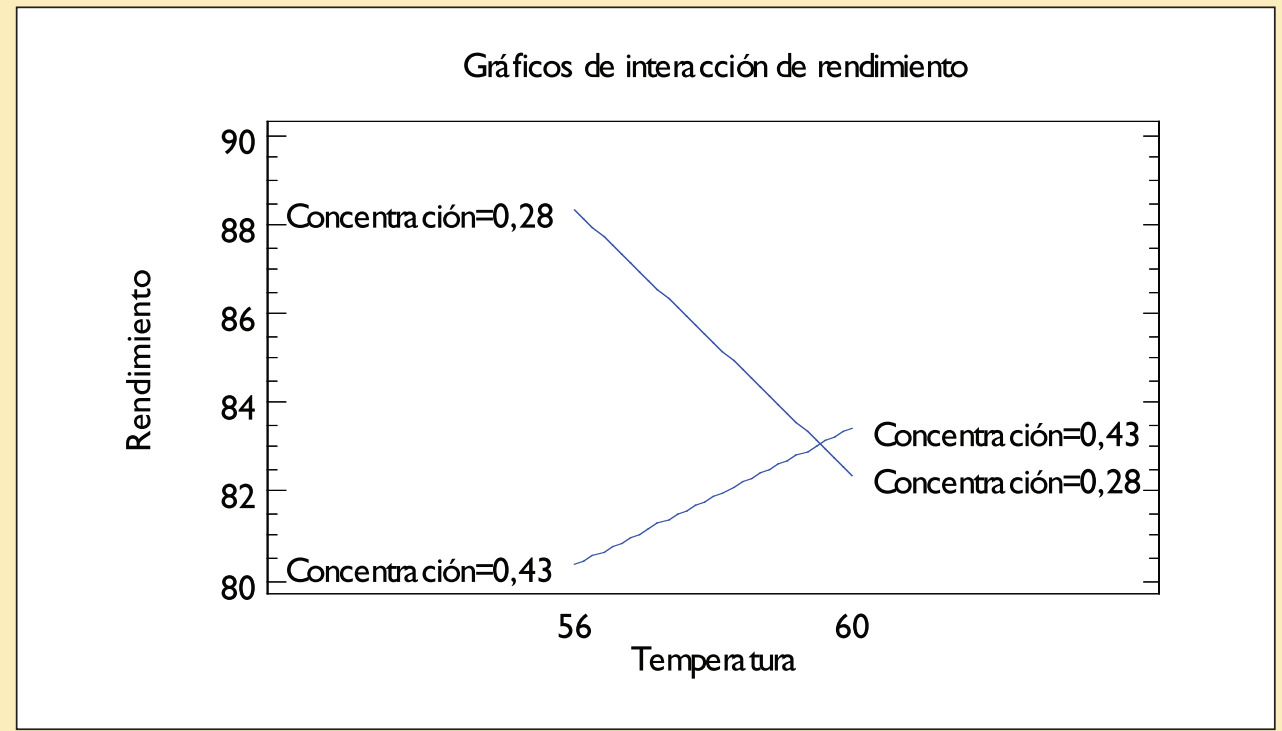

Figura 3. Interacción del rendimiento en función de la temperatura.

A través de la figura 4 se puede determinar que la temperatura apropiada esta alrededor de $59^{\circ} \mathrm{C}$ y una concentración del 0,35-0,36 para alcanzar rendimientos alrededor del $90 \%$.

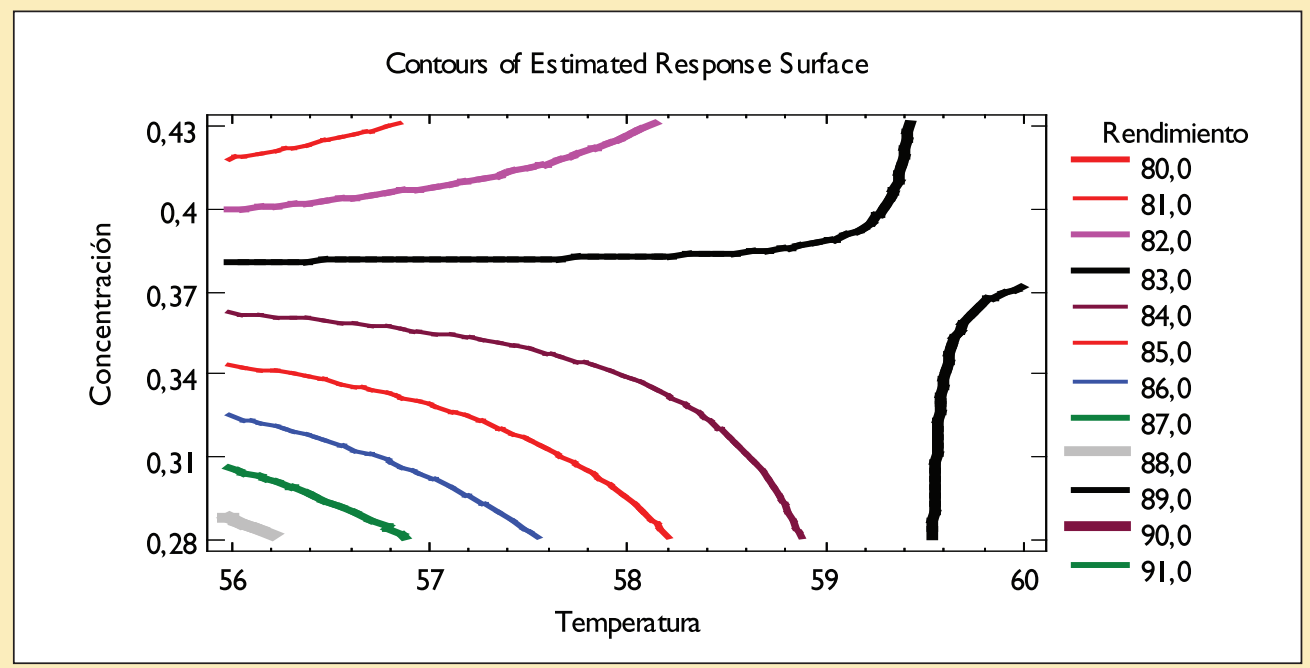

Figura 4. Rendimiento de la reacción. 


\subsection{Característica y funcionalidad del Biodiesel}

En esta tercera fase, se procedió a determinar la composición del biodiesel que es mezcla de ester metílico de ácidos grasos en un $96 \%$ (según el Infor- me de análisis del CIBAVI), además de determinar ciertas propiedades del biodiesel al compararlas con las normativas internacionales a $40^{\circ}$, presentadas en la Tabla 4.

Tabla 4. Características del Biodiesel

\begin{tabular}{llll}
\hline Características & Unidad & \multicolumn{2}{c}{ Valores } \\
\cline { 3 - 4 } & & grasa & norma \\
\hline Punto de inflamación & ${ }^{\circ}$ C & 134 & $>130$ \\
Viscosidad cinética & cSt a & 4 & $1,9-6$ \\
Poder calorífico & BTU/Lbs & 16034 & \\
\hline
\end{tabular}

Finalmente se procedió a utilizar este combustible mezclando con el diesel en una relación de 5$10-20 \%$ y se aplicó en un motor de diesel, el mismo que funcionó normalmente caracterizándose por humos de color blanco, a diferencia de los humos de diesel de color oscuro. Posteriormente, se analizaron las emisiones de combustión a través de un analizador de gases, de marca Ecoline 4000, estableciéndose una disminución tanto en la emisión de $\mathrm{SO}_{2}$ como de $\mathrm{CO}_{2}$, pero incrementando el porcentaje en $\mathrm{NO}_{2}$ conforme aumentaba la relación biodiesel-diesel.A continuación, se muestra el comportamiento del $\mathrm{SO}_{2}$ y del $\mathrm{NO}_{2}$ respectivamente. (Figuras 5 y 6 ).

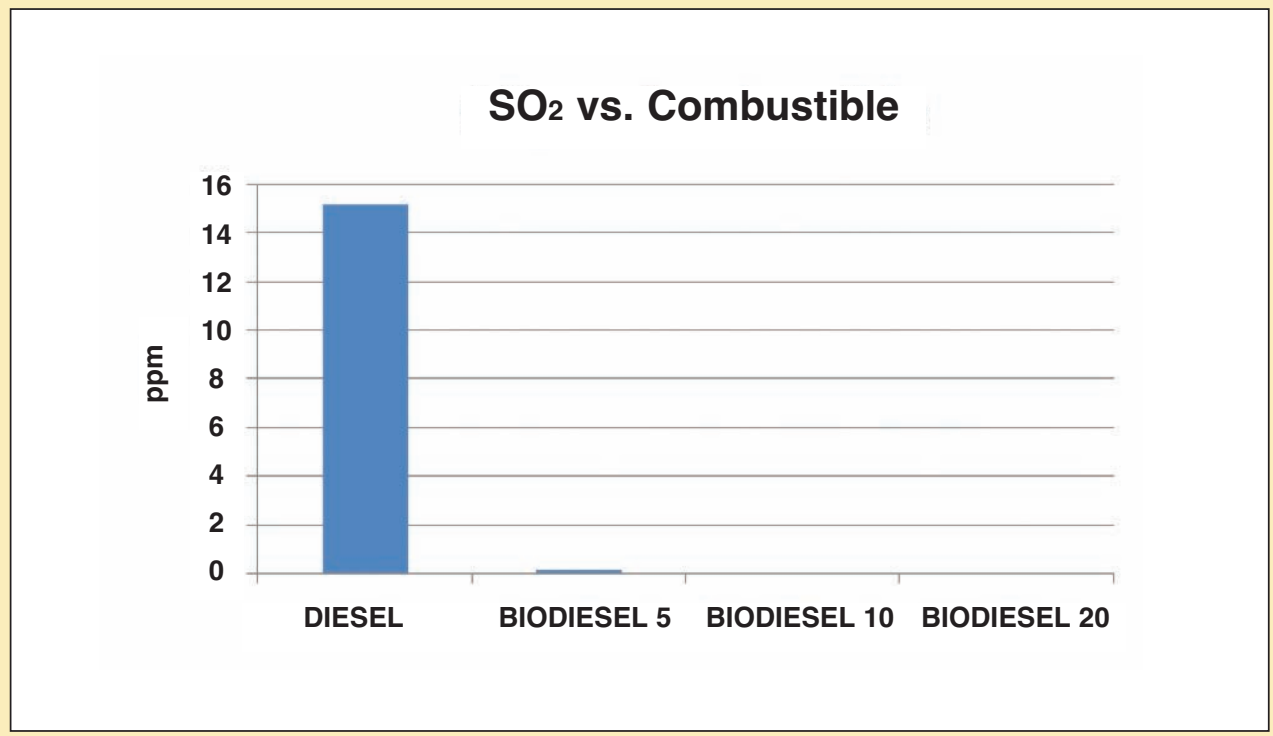

Figura 5. Comportamiento del $\mathrm{SO}_{2}$ en función de la adición del biodiesel. 


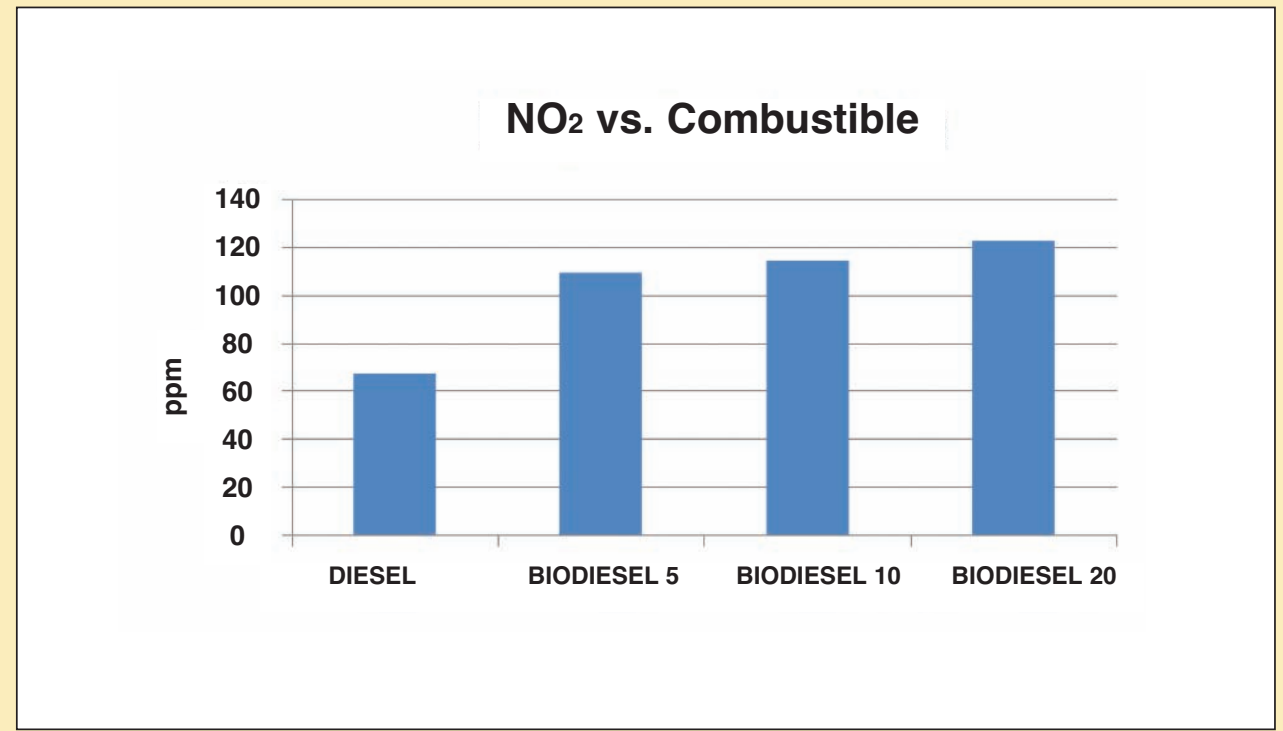

Figura 6. Comportamiento del $\mathrm{NO}_{2}$ en función de la adición del biodiesel.

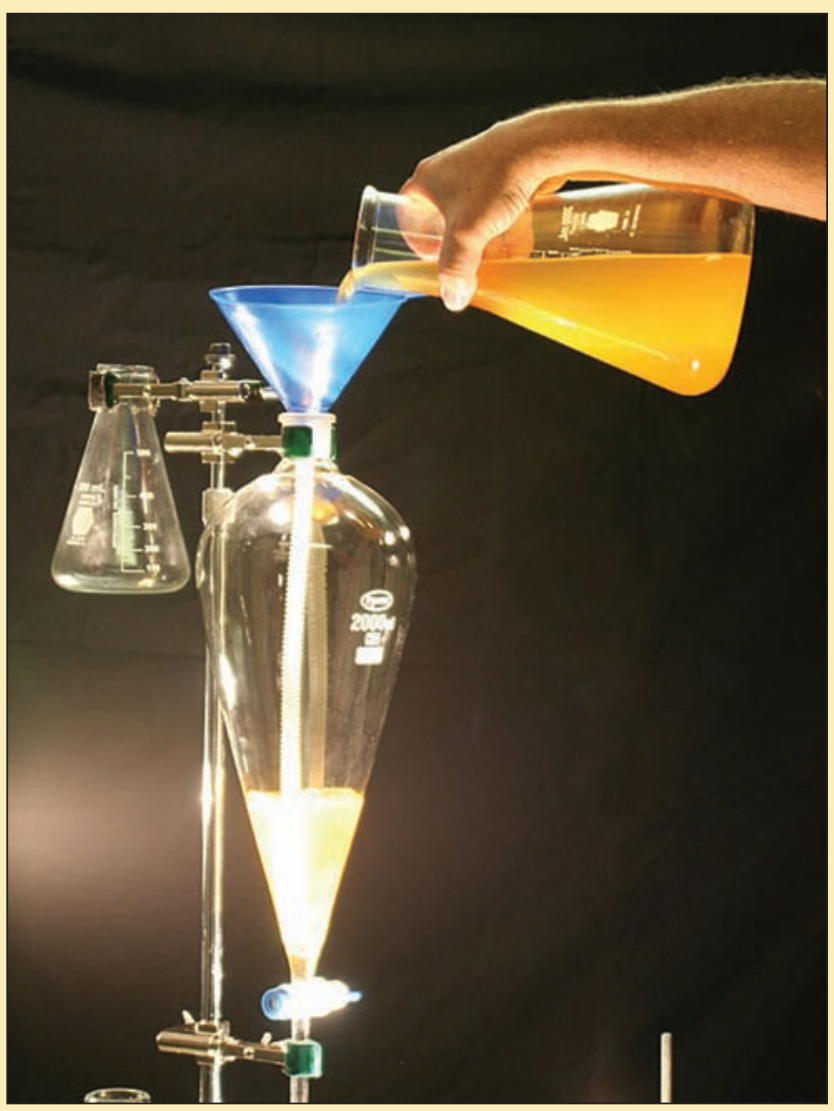

\section{Conclusiones}

Se observa experimentalmente que la conversión de triglicéridos es elevada cuando el catalizador tiene concentraciones de entre 2 y $3 \mathrm{~g}$, considerando que con estos valores no se requiere de un proceso de separación ni lavados.

De la optimización de la reacción, llevada a cabo mediante un diseño factorial de experimentos en el que se estudió la influencia de la concentración inicial de catalizador y de la temperatura sobre el rendimiento de la reacción, se determinó que la temperatura de reacción y la concentración de catalizador tienen un efecto negativo sobre el rendimiento de la fase éster secundaria de saponificación de triglicéridos.

Se consideran como óptimas las siguientes condiciones de operación ya que con ellas se obtiene un rendimiento entre 88 y el $90 \%$ : concentración de catalizador de 3,7 a $3,8 \%$ a una temperatura de $58^{\circ} \mathrm{C}$. Con esas condiciones no es necesario el proceso de lavado y purificación del biodiesel.

Además, se determinó que el biodiesel obtenido cumple con los estándares internacionales y que disminuye las emisiones tanto de $\mathrm{SO}_{2}$ como el $\mathrm{CO}_{2}$ pero aumenta la emisión de $\mathrm{NO}_{2}$. 


\section{Agradecimiento}

El principal agradecimiento es hacia las autoridades de la Universidad Politécnica Salesiana por haber brindado su apoyo e impulso hacia esta investigación.

\section{Referencias}

Escalona, García e Hidalgo. 1998. Química Orgánica s.I. Edit.Pentice Hall. Ecuador.

Gutierrez-De la Vara, R. 2004. Análisis y Diseño de
Experimentos. Ed. McGraw-Hill.

Montero, A. 2002 (2003). Estudio de Transesterificación de aceite Vegetal con Metanol, Escuela Superior de Escuelas Esperimentales. Lima, Perú, Pp. 22-23.

Pedron Agudelo, John, S., Cano. 2004. Estudio Experimental De Las Variables Que Afectan la Reacción de Benjumea. Revista Scientia et Technica. No. 24, Mayo 2004 , Vol. X.

Sanz, C. 1967. Enciclopedia de la carne. Espasa-Calpe S.A, Madrid, España.

Tempelton. 1983. Química Orgánica. Paraninfo.
Se determinó que el biodiesel obtenido cumple con los estándares internacionales y que disminuye las emisiones tanto de $\mathrm{SO}_{2}$ como de $\mathrm{CO}_{2}$ pero aumenta la emisión de $\mathrm{NO}_{2}$.

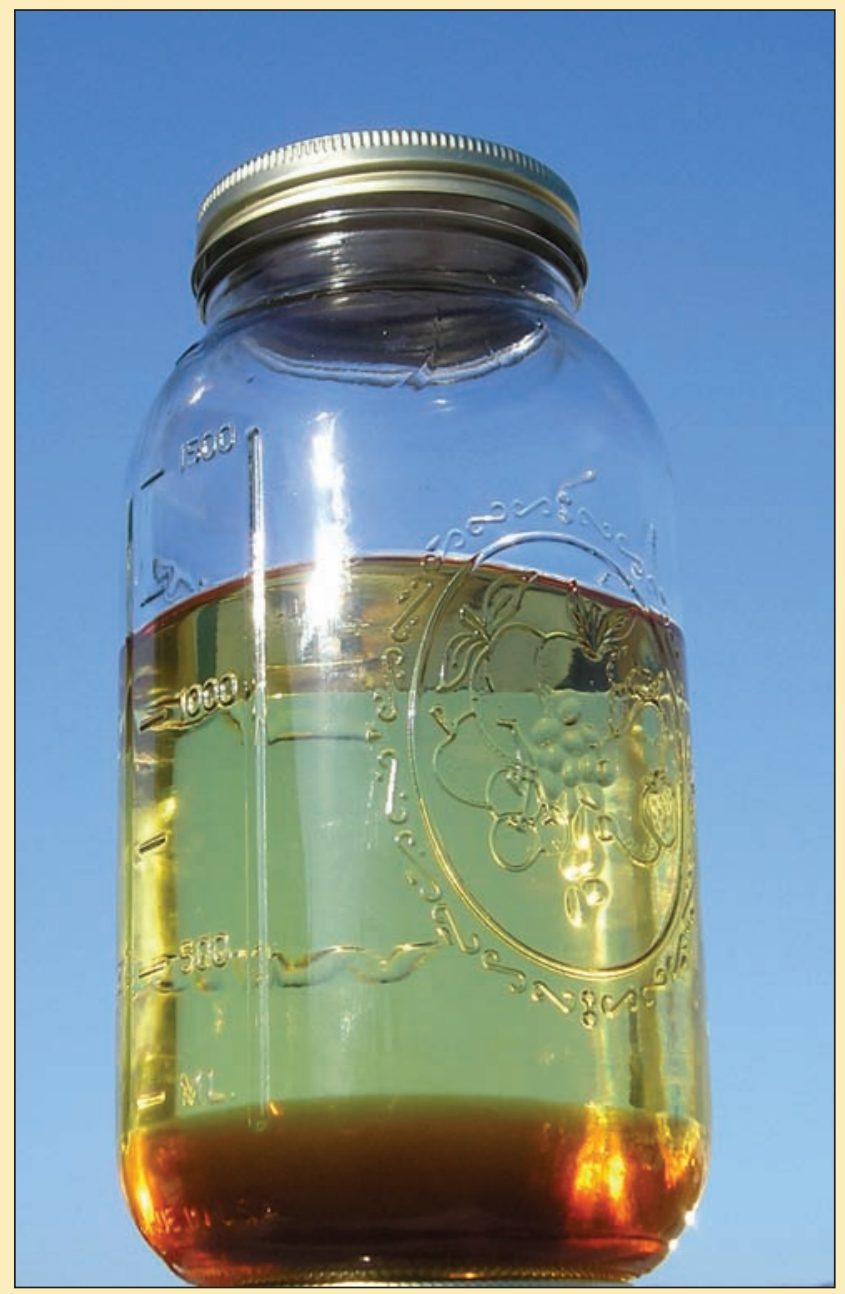

